\title{
The Shape of Confined Polymer Chains
}

\author{
A. SIKORSKI* \\ Department of Chemistry, University of Warsaw \\ Pasteura 1, 02-093 Warsaw, Poland
}

(Received December 9, 2002)

\begin{abstract}
The linear polymer chains were modeled on a simple cubic lattice. The excluded volume was included into the model while the system remained athermal (no attractive interactions) which simulated the good solvent conditions. The polymer chain was located between two parallel impenetrable walls and the distance between the walls was changing. No interaction between walls and polymer segments was assumed. These models of polymer chains were simulated by the means of the Monte Carlo method. In the sampling algorithm we used the micromodifications of chain's conformations to sample efficiently the conformational space. The size of the chain did not change monotonically for all lengths under consideration (up to 800 statistical segments). For distances between the plates close to the double value of chain's radius of gyration the size of the chain approached its minimum value. It was shown that scaling of chain dimensions with its length changed from $N^{1.18}$ to $N^{1.5}$ while the distance between the walls was decreasing. The behavior of the asymmetry of the chain was found to be analogous to that of the radius of gyration.
\end{abstract}

PACS numbers: 02.50.Ng, 05.10.Ln, 61.25.Hq

\section{Introduction}

Polymer chains confined in a slit formed by solid surfaces should be treated as an important phenomenon due to its practical applications like chromatography, colloidal stabilization, lubrication, etc. [1]. The confinement of polymer chains is also interesting from the theoretical point of view because the presence of the impenetrable surfaces usually changes dramatically the properties of such chains when compared with the case of free chain in solution. Theoretical considerations concerning the confined macromolecules go back to the works of de Gennes [2].

*e-mail: sikorski@chem.uw.edu.pl 
The theoretical model and the results are limited to well-defined geometries of the confinement like a slit or a cylinder only.

Computer simulations are also very useful in studying confined polymer chains because of the difficulties with analytical solutions as well as experiments of such systems. Among many works devoted to this subject only a few of them concerned static properties of single chains. Van Vliet and ten Brinke carried out Monte Carlo simulations of lattice models of polymer chains [3]. They found that the size of a polymer chain exhibits a universal behavior disregarding its length. They also discussed the problem of orientation of the chain while squeezing the walls. Milchev and Binder built an off-lattice model of a polymer chain and studied it by the means of the Monte Carlo method [4]. Besides, the results concerning the size of a chain they also calculated the polymer density profiles inside the slit. Cifra and Bleha employing lattice model checked the influence of kind of the confinement (slit versus cylinder) on the properties of a polymer chain [5]. Arteca studied the grafted polymer chain between two walls (so-called "polymer mushrooms") [6]. He found that the properties of a squeezed chain could depend on its initial conformation in unconfined state taking into account the number of entanglements. Recently, some intensive simulations concerning lattice models of single star-branched polymers were done [7]. It was shown that besides the universal behavior of the polymer size, the similar results were found for the short-time (relaxation processes) and long-time (self-diffusion) dynamic properties. The possible changes in the mechanism of a chain motion during the squeezing of walls were discussed there.

In this work we presented results of calculations concerning some static properties of single linear lattice polymers confined between two parallel impenetrable walls. We have chosen a simple cubic lattice because the properties of free polymer chains were frequently and precisely determined and, therefore, we had a good reference state (unconfined chain). The chains were simulated with the exclude volume only and with no attractive segment-segment and segment-wall interactions which corresponded to good solvent conditions. The Monte Carlo simulation algorithm was previously worked out for all chain topologies and was used for some calculations of different conditions like polymer melts, adsorbed chains, grafted chains [7-9]. The classical version of the algorithm was chosen because a new multi-bond algorithm was inefficient in the confined space.

\section{Model and simulation algorithm}

The locations of polymer segments were restricted to the vertex of a simple cubic lattice in order to speed up the simulations. The lattice approximation was sufficient for our purposes as we studied parameters describing the entire chain. There was no distinction between interaction of segment-segment, segment-solvent, and segment-surface and, therefore, the system should be considered as athermal. The only potential used was the excluded volume which can 
be easily realized by forbidding the double occupancy of lattice sites by polymer segments. This condition reproduced good solvent conditions where the excluded volume was not compensated by the attraction between polymer segments. Chains were flexible because no conformational biases were introduced. All the calculations were performed for single linear chains (infinite diluted solution). The chains were put in a Monte Carlo box with periodic boundary conditions set in $x$ and $y$ directions only. The size of the Monte Carlo box, $L=200$ lattice units, was large enough to minimize the influence of its size on the properties of the system. The walls that confined the polymer chain were put to be parallel to the $x y$ plane. This surface was impenetrable for polymer segments which was realized by forbidding polymer segments to move to the latticed points with non-positive $z$-coordinates. One surface was located at the plane $z=0$ while the second one at the plane $z=d+1$. This implied that in the $z$-direction there were $d$ consecutive lattice sites that can be occupied by polymer beads. Figure 1 shows a schematic representation of a chain located between the walls.

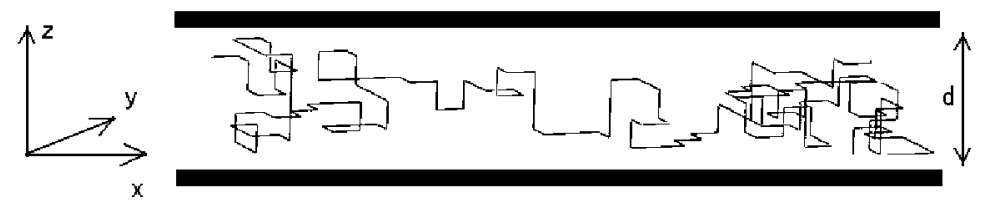

Fig. 1. The scheme of a linear polymer chain trapped between two parallel impenetrable walls. The surfaces are located $d$ apart.

The Monte Carlo algorithm used was based on the classical Metropolis scheme. The conformation of a polymer chain underwent a series of local micromodifications. The following set of micromodifications was previously worked out for simulation cubic lattice models: two-bond motion, three-bond motion, three-bond crankshaft motion and chain's ends reorientations [10]. A new conformation obtained after such micromodification was accepted due to topological constraints and the excluded volume condition. The series of local changes of conformation were selected at random along the chain during the simulation process. The Monte Carlo simulation run consisted of $10^{7}-10^{8}$ time units (a time unit corresponds to an attempt of all micromodifications per polymer segment). Before the actual simulation run, an equilibration run was always performed. The equilibration run consisted of $10^{6}$ time units. One has to remember that for small distances between the walls and for longer chains algorithm was much less efficient. In order to guarantee the proper sampling of different parts of the phase space for each model chain at a given distance between the walls, the simulation was carried out 20-30 times starting from quite different conformations. The very important question concerning the entanglements in a confined polymer chain was recently raised by Arteca [6]. He argued that the initial conformation of the chain in the 
confinement could influence chain properties. To avoid such bias we started simulations from two quite different pools of initial states. The first one was a random conformation built as self-avoiding walks for surfaces with the shortest distance $(d=3)$. Then, the next simulation runs were made and the distance $d$ was gradually increasing. The second starting pool of conformations contained unconfined ("free") chains and in the next simulation runs the walls appeared and distance between them was gradually diminishing.

\section{Results and discussion}

The simulations were carried for linear chains consisting of $N=50,100$, 200 , and 800 beads. The changes of the distance between the impenetrable surfaces $d$ were done basing on the data obtained recently for the similar model of star-branched polymers [7]. It this paper it was shown for which distances $d$ the changes of static properties of branched polymers were pronounced and interesting. As our linear chains had almost the same lengths as these branched polymers, the changes of the distance between plates were made in the similar range. This parameter was changed from $d=3$ (the smallest possible value for which the micromodifications of polymer conformations can be made) to $d=50$ (the distance slightly higher than the mean diameter of the longest chains under consideration).

The first problem to be discussed is the dependence of the confinement on the size of a polymer chain. The most general parameter that describes the size of a polymer is the mean-square radius of gyration $\left\langle S^{2}\right\rangle$ defined as:

$$
S^{2}=\sum_{i=1}^{N}\left[\left(x_{\mathrm{cm}}-x_{i}\right)^{2}+\left(y_{\mathrm{cm}}-y_{i}\right)^{2}+\left(z_{\mathrm{cm}}-z_{i}\right)^{2}\right],
$$

where the subscript 'cm' denotes the center-of-mass. Figure 2 presents the logarithmic dependence of the $\left\langle S^{2}\right\rangle$ parameter on the number of polymer segments $N$ for some distances between the surfaces $d$. For the sake of comparison we included the data concerning the size of a free chain (unconfined) into this plot. Every parameter describing the size as the radius of gyration should scale as $\left\langle S^{2}\right\rangle \sim N^{\gamma}$, where $\gamma$ is an exponent that depends on the dimensionality of space only [2] and this behavior is clearly seen on the plot. The scaling exponent for the free chain $\gamma=1.19$, which is very close to theoretical predictions, where $\gamma=1.18$ [2]. For larger values of the distance $d$ the exponent is slightly smaller than for the free chain: $\gamma=1.13$. This behavior will be discussed and explained below. The further decreasing of the distance between the surfaces leads to the changes in the scaling behavior. One can observe that for the intermediate distances $(d=10)$ there are two regimes of scaling: for shorter chains the scaling exponent $\gamma=1.19$ (as for unconfined chains) while for longer chains $\gamma=1.38$ (closer to 1.5 like for two-dimensional chains). This case will also be discussed below. For small distances $(d=3)$ the scaling exponent $\gamma=1.50$, which is exactly the same value as theoretically predicted and 
found experimentally for two-dimensional polymer structures (like thin polymer adsorbed films) [2]. This last result will become obvious if one can imagine chains squeezed along the $z$-axis to the layer of thickness 3 while along the other directions their size is two orders of magnitude larger. This means that the chain is actually two-dimensional.

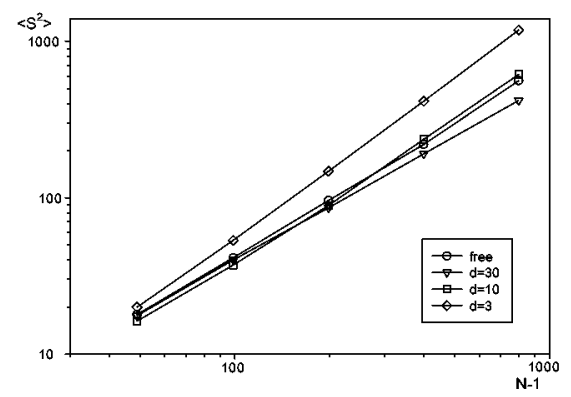

Fig. 2. The mean-square radius of gyration $\left\langle S^{2}\right\rangle$ versus the distance between the walls for some chain lengths. The values of distance $d$ are given in the inset.

Besides the differences in the scaling behavior one can notice from Fig. 2 the interesting changes of the radius of gyration when the distance between the impenetrable walls diminishes: the size of the polymer chain initially decreased and then started to increase when the surfaces are approaching each other. This behavior is better visible in Fig. 3, where we have plotted the mean-square radius of gyration as a function of the distance $d$ for some chain lengths. It can be observed that while squeezing the walls the size of the chain slightly decreases which was expected. But after the certain distance $d^{*}$ was approached, the size of chain starts to grow rapidly. The shape of the radius of gyration dependence on the distance between the walls is the same for all chain lengths under consideration. The main difference is the location of the mimima denoted as $d^{*}$, which are shifted towards higher values of $d$ for longer chains. The same behavior was found for other models of confined linear polymer [3,5] and recently for lattice star-branched chains [7]. The explanation of this phenomenon seems to be simple: the diameter of a chain can be estimated as $2\left\langle S^{2}\right\rangle^{1 / 2}$ and the point $d^{*}$ is located near this value. This suggests that the real squeezing of a chain starts below $2\left\langle S^{2}\right\rangle^{1 / 2}$ and is realized by a transition to an almost two-dimensional chain.

Knowing the type of the chain conformation's changes one can go back to the analysis of the values of scaling exponents $\gamma$. Based on the dependence of the radius of gyration on the distances between the walls discussed above, we know that the chains of various lengths are simply in quite different states for a given value of $d$. For example, at $d=30$ (see Fig. 3) a chain with $N=49$ beads is almost unaffected $\left(d^{*}=8.5\right)$, while a chain with $N=800$ is already compressed $\left(d^{*}=48\right)$. This behavior is even better visible for the case $d=10$, where one 

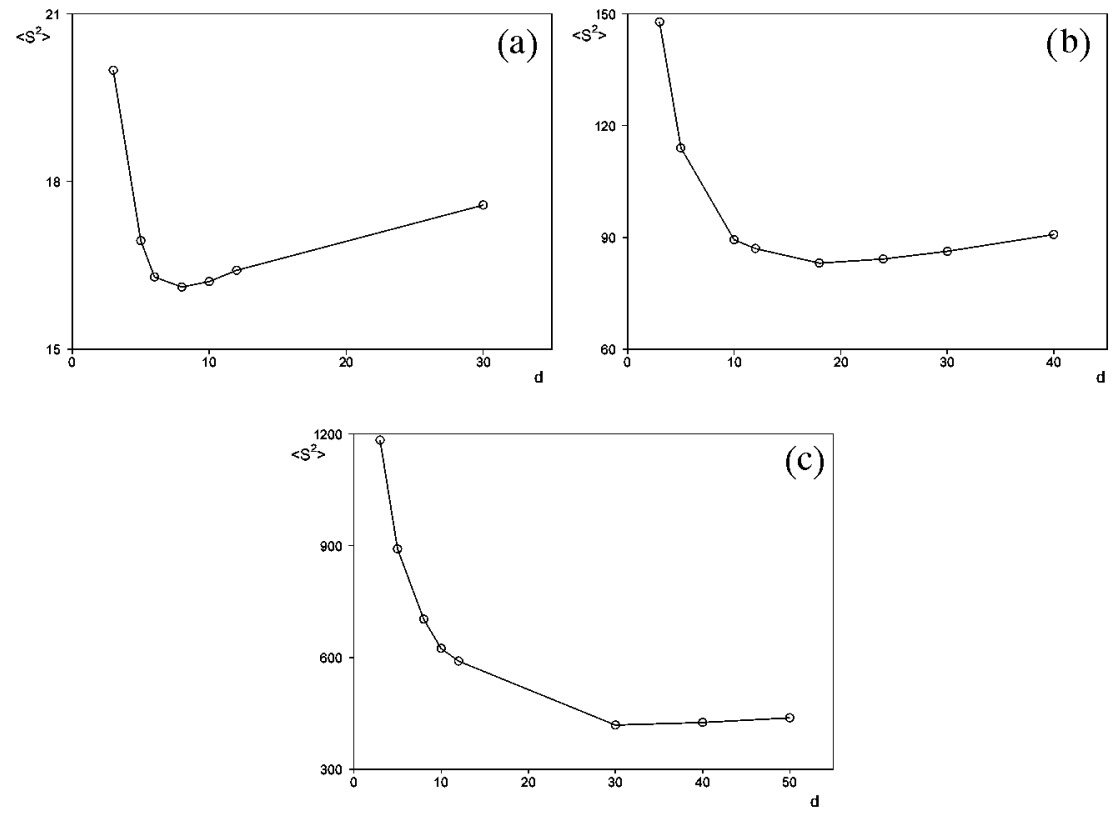

Fig. 3. The mean-square radius of gyration $\left\langle S^{2}\right\rangle$ versus the distance between the walls for $N=50$ (a), $N=200$ (b), and $N=800$ (c).

can distinguish two different scaling regimes (Fig. 2). The properties of confined linear chains discussed above can be compared with those of star-branched chains. There are no qualitative differences in the changes of the radius of gyration with the size of slit for both linear and star-branched chains. The main difference is the location of minima on the $S^{2}(d)$ curve: stars are considerably smaller than linear chains and thus their $d^{*}$ is also smaller.

The transition from a three-dimensional to a two-dimensional polymer system is not sharp but continuous. This transition is not located on the minimum of the radius of gyration curve but somewhere below this point. The analysis of other size parameters does not give much insight into the characteristics of the transition, either. Figure 4 shows the plot of the ratio $\left\langle S^{2}\right\rangle /\left\langle R^{2}\right\rangle$, where $\left\langle R^{2}\right\rangle$ is the mean-square end-to-end distance of a polymer chain. Going down with the distance $d$ this parameter slightly increases from values close to theoretical 0.157 for free chains with the excluded volume to those close to 0.167 (a value characteristic for random flight chains) at $d^{*}$ [2]. The further squeezing leads to the rapid decreases of this ratio. The behavior of this ratio and of similar parameters can be treated as an additional criterion for finding and characterizing the changes of chain's conformations [11].

The distribution of polymer segments inside the slit can also be studied in our model. Figure 5 presents segment density profiles for some distances between the walls. The density profile is symmetric with respect to the walls for all cases. The 


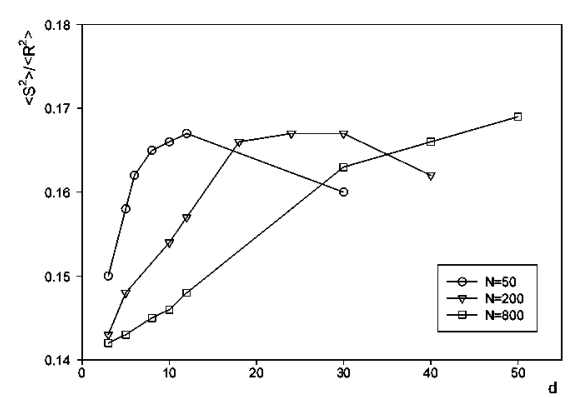

Fig. 4. The ratio $\left\langle S^{2} / R^{2}\right\rangle$ as a function of the distance between walls $d$ for some chain lengths. The values of chain lengths $N$ are given in the inset.

similar effect was observed by Milchev [12] for diluted solution of linear chains. For a small slit $(d=3)$ with squeezed polymers there is no difference in density profiles for different chain lengths. In the case of slightly deformed polymers $\left(d>d^{*}\right)$ the distribution changed its character, i.e. a plateau appears in the middle part of the slit. This is visible in Fig. 5 for chains $N=50$ at $d=30$.
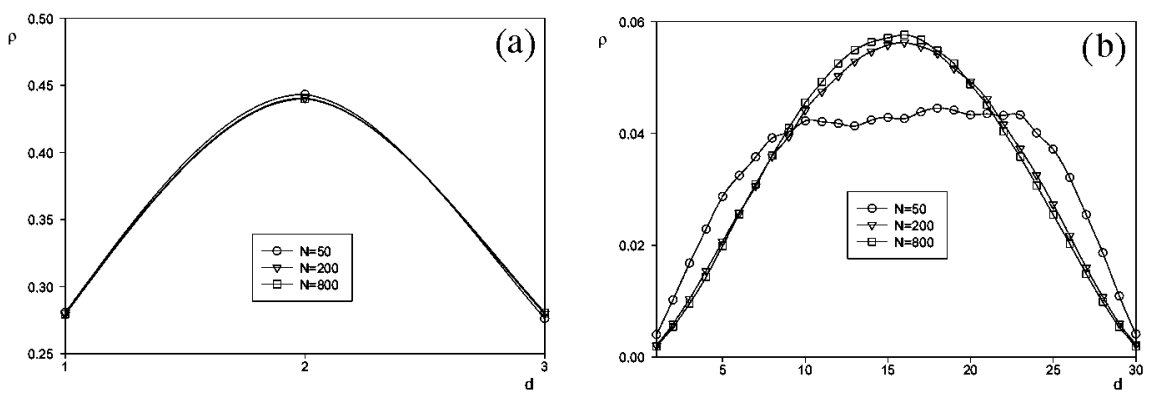

Fig. 5. The density of polymer segments $\rho$ as a function of the distance $d$ between walls for $d=3$ (a) and $d=30$ (b). The values of chain lengths $N$ are given in the inset.

The last question concerns the real influence of the walls, i.e. changes of the shape of the chain under consideration. The instantaneous shape of a polymer chain can be also derived from the simulation results. We can define a tensor:

$$
S=\left|\begin{array}{ccc}
S_{x x} & S_{x y} & S_{x z} \\
S_{x y} & S_{y y} & S_{y z} \\
S_{x z} & S_{y z} & S_{z z}
\end{array}\right|,
$$

which is built from the following elements:

$$
S_{x y}=\sum_{i=1}^{N}\left(x_{\mathrm{cm}}-x_{i}\right) \cdot\left(y_{\mathrm{cm}}-y_{i}\right), \quad \text { etc. }
$$


Comparing the above tensor with the definition of the radius of gyration in Eq. (1) one can see that the trace of $\boldsymbol{S}$ is equal to the squared radius of gyration:

$$
S^{2}=\operatorname{tr}(\boldsymbol{S})=S_{x x}+S_{y y}+S_{z z} .
$$

The diagonalization of the tensor $\boldsymbol{S}$ gives us eigenvalues $L_{1}^{2}, L_{2}^{2}, L_{3}^{2}$, which correspond to the three main axes of the equivalent ellipsoid. One can describe the shape of the chain by giving the ratio of these axes. The following approximate ratio was given for the linear random flight chains (without the excluded volume) on a simple cubic lattice by the means of the Monte Carlo simulations [13]:

$$
L_{1}^{2}: L_{2}^{2}: L_{3}^{2}=11.7: 2: 7: 1
$$

This result means that a polymer coil does not have the spherical shape but it has a bean-like shape. The operations on the above ratios are rather inconvenient. Therefore, Rudnick and Gaspari introduced the asphericity factor $\delta$ [14]:

$$
\delta=\frac{\left\langle\left(L_{1}^{2}-L_{2}^{2}\right)^{2}+\left(L_{1}^{2}-L_{3}^{2}\right)^{2}+\left(L_{2}^{2}-L_{3}^{2}\right)^{2}\right\rangle}{2\left\langle\left(L_{1}^{2}+L_{2}^{2}+L_{3}^{2}\right)^{2}\right\rangle} .
$$

This parameter was designed in order to take the value 0 for the pure spherical shape and 1 for a one-dimensional rod.

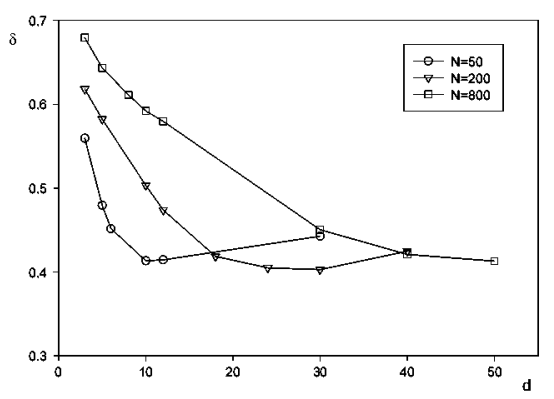

Fig. 6. The asphericity factor $\delta$ as a function of the distance between walls $d$ for polymer chains with $N=50,200$, and 800 segments.

Figure 6 presents the dependence of the asphericity factor $\delta$ on the distance between the surfaces for some chain lengths. The asphericity of a single 'free' linear chains was found to be $\delta=0.44$ which is very close to the theoretical predictions $\delta=0.53[13,14]$. Bringing surfaces together has the similar influence on the asphericity factor to the radius of gyration. Initially, (above $d^{*}$ ) the asymmetry of a confined chain does not increase but even slightly decrease. The further squeezing of walls leads to the considerable increasing of asymmetry which can be explained by the forming of two-dimensional structure. The chain's asymmetry for the lowest size of the slit $(d=3)$ increases with the length of the polymer: $\delta=0.56$ for $N=50$ and $\delta=0.68$ for $N=800$. This behavior of the polymer's shape can be explained by the fact that the chain for slit larger than $d^{*}$ is rather reoriented 
than deformed [2]. The reorientation means that principal axis of the chain starts to place parallel to the walls.

The main conclusion from this work is that the presence of the confinement has a huge impact on its size and shape. The behavior of linear chains and star-branched polymers is qualitatively the same in the same conditions. Further studies should contain dynamic properties of confined linear chains and thermodynamical description of the transition from a three-dimensional chain to a two-dimensional chain.

\section{References}

[1] R. Eisenriegler, Polymers near Surfaces, World Scientific, Singapore 1993.

[2] P.-G. de Gennes, Scaling Concepts in Polymer Physics, Cornell University Press, Ithaca 1979.

[3] J.H. van Vliet, G. ten Brinke, J. Chem. Phys. 93, 1436 (1990).

[4] A. Milchev, K. Binder, Eur. Phys. J. B 3, 477 (1998).

[5] P. Cifra, T. Bleha, Macromol. Theory Simul. 8, 603 (1999).

[6] G. Arteca, Macromol. Theory Simul. 8, 137 (1999).

[7] A. Sikorski, P. Romiszowski, J. Chem. Phys. 116, 1731 (2002).

[8] A. Sikorski, P. Romiszowski, Acta Phys. Pol. A 100, 477 (2001).

[9] A. Koliński, M. Vieth, A. Sikorski, Acta Phys. Pol. A 79, 601 (1991).

[10] A. Sikorski, Makromol. Chem. Theory Simul. 2, 309 (1993).

[11] P. Romiszowski, A. Sikorski, Comput. Theor. Polym. Sci. 11, 129 (2001).

[12] A. Milchev, Eur. Phys. J. E 8, 531 (2002).

[13] G. Zifferer, J. Chem. Phys. 109, 3691 (1998).

[14] J. Rudnick, G. Gaspari, J. Phys. A 19, L191 (1986). 